\title{
Synthesis, antimalarial activity, and docking studies of monocarbonyl analogues of curcumin
}

\author{
Amina S. YUSUF, ${ }^{1}$ Ibrahim SADA,${ }^{1}$ Yusuf HASSAN,${ }^{1, *}$ Temitope O. OLOMOLA,${ }^{2}$ Christiana M. ADEYEMI, ${ }^{3}$ \\ and Sunday O. AJIBADE ${ }^{4}$ \\ ${ }^{1}$ Department of Chemistry, Umaru Musa Yar'adua University, Katsina, Nigeria \\ ${ }^{2}$ Department of Chemistry, Obafemi Awolowo University, Ile-Ife, Nigeria \\ ${ }^{3}$ Department of Chemistry, Mountain Top University, Mowe, Nigeria \\ ${ }^{4}$ Department of Chemistry, Plateau State University, Bokkos, Nigeria
}

\begin{abstract}
The synthesis of five monocarbonyl analogues of curcumin is described. In vitro anti-malarial assay of the compounds was carried out and the effect of the substituents on the aryl ring has been described. The results show that all the five compounds exhibited some reasonable activity against the chloroquine-resistant plasmodium parasite. Molecular docking studies further confirmed the observed biological activity of the compounds.
\end{abstract}

Keywords: curcumin, monocarbonyl analogues, antimalarial activity, docking studies.

\section{Introduction}

Malaria is one of the world's most endemic diseases, affecting over 100 tropical and sub-tropical countries. Plasmodium falciparum remains the most dangerous of the five plasmodium species to man. In 2013, there were approximately 198 million cases of malaria, and estimated number of 584,000 deaths mainly in the African region [1]. Curcumin is a bis-benzylidine compound isolated from Curcuma longa L (Figure 1) which demonstrates an incredible potential as a lead compound [2-6]. However, the presence of the methylene group in its structure confers certain instability to the compound [7]. Consequently, a number of disadvantages in pharmacokinetics such as poor bioavailability and need for continuous use limits its application $[8,9]$. Thus, more efforts are now directed toward the synthesis and biological assays of the monocarbonyl analogues, and preliminary results reveals that the compounds possess anticancer [10], antibacterial [11], anti-Cushing's disease [12], antifungal [13], anti-inflammatory [14], antitrypanosomal and Leishmania [15], and antimalarial activities [16]. In this work, a series of known monocarbonyl curcumin analogues are synthesized and evaluated for antimalarial activity among which the antimalarial activity of two have already been previously reported [16]. But as a proof of concept, it was decided to reassess them along with the other ones. Also, the computational studies were explored to further understand the interaction of the compounds with the target protein, a contribution that has not been reported for all the compounds.

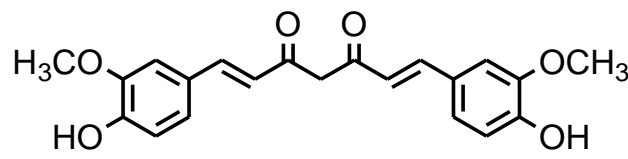

Figure 1. Chemical structure of curcumin

\section{Experimental}

\subsection{Materials and methods}

All chemicals and solvents were purchased from Aldrich and used as received. ${ }^{1} \mathrm{H}$ and ${ }^{13} \mathrm{C}$ NMR spectra were recorded on a Bruker AVANCE 400 spectrometer. IR spectra were recorded on a Perkin-Elmer FT-IR Spectrum BX spectrophotometer. Melting points were determined on an Electrothermal melting point apparatus and are uncorrected. Analytical TLC was carried out using precoated silica gel 60 F254 plates $(0.2 \mathrm{~mm}$ thickness, Merck).

\subsection{Synthesis}

General Procedure for the preparation of analogues $3 a$, $\boldsymbol{b}($ Method A)

To a stirred solution of aromatic aldehyde $(20 \mathrm{mmol}, 2$ equiv.) and acetone (10 mmol, 1 equiv.) in $15 \mathrm{ml}$ of ethanol at $-5{ }^{\circ} \mathrm{C}$ was added $\mathrm{CF}_{3} \mathrm{CO}_{2} \mathrm{H}(10 \mathrm{ml})$ drop wise over 30 minutes. The solution was then allowed to warm up to room temperature and left to stir for $10 \mathrm{~h}$. On addition of dilute $\mathrm{HCl}$, a precipitate is formed, which is then filtered and recrystallized to give the desired product.

Compound $3 a$ : Yellow powder, $82 \%$ yield, $\mathrm{mp} 86-88^{\circ} \mathrm{C}$. ${ }^{1} \mathrm{H},{ }^{13} \mathrm{C}$ NMR and IR spectra agreed with the literature [14b].

Compound $3 \boldsymbol{b}$ : Yellow powder, $100 \%$ yield, mp 241$244^{\circ} \mathrm{C} .{ }^{1} \mathrm{H},{ }^{13} \mathrm{C}$ NMR and IR spectra agreed with the literature [7].

General Procedure for the preparation of analogues $3 c$, $\boldsymbol{d}, \boldsymbol{e}(\operatorname{Method} B)$

The same procedure as in method A except that $40 \%$ $\mathrm{NaOH}$ solution in water was substituted for the $\mathrm{CF}_{3} \mathrm{CO}_{2} \mathrm{H}$.

Compound 3c: Yellow powder, $81 \%$ yield, mp 260$265^{\circ} \mathrm{C} .{ }^{1} \mathrm{H},{ }^{13} \mathrm{C}$ NMR and IR spectra agreed with the literature [17].

\footnotetext{
* Corresponding author. E-mail adress: yusuf.hassan@umyu.edu.ng (Yusuf Hassan)
} 
Compound 3d: Yellow powder, 83\% yield, mp 131$134{ }^{\circ} \mathrm{C} .{ }^{1} \mathrm{H},{ }^{13} \mathrm{C}$ NMR and IR spectra agreed with the literature [10c].

Compound 3e: Yellow powder, 85\% yield, mp 212$215^{\circ} \mathrm{C} .{ }^{1} \mathrm{H},{ }^{13} \mathrm{C}$ NMR and IR spectra agreed with the literature [10c].

\subsection{Antimalarial assay}

A test tube containing 5\% parasitaemia was charged with a test solution $(0.1 \mathrm{ml})$ and the culture medium $(0.2 \mathrm{ml})$ and mixed thoroughly. The sensitivity of the parasites to each test compound with the serial concentrations of 10 , $5,2.5$, and $1.25 \mathrm{mg} / \mathrm{ml}$ was determined under microscope at $37{ }^{\circ} \mathrm{C}$ after $24 \mathrm{~h}, 48 \mathrm{~h}$ and $72 \mathrm{~h}$ of incubation. In order to supply the $95 \%$ of nitrogen, $2 \%$ of oxygen and $3 \%$ of carbon dioxide necessary for the incubation, the test tubes were all transferred into a jar containing a burning candle and then replaced the cover until the flame stopped burning. The preparation of the control groups was performed by mixing the culture media with the positive erythrocytes to serve as the negative control. While the culture media plus positive erythrocytes and artemether (a known antimalarial) as the positive control [18].

At the end of 24,48 , and $72 \mathrm{~h}$ incubation periods, an aliquot of each culture medium was smeared on a slide, stained using Geimsa staining technique and then observed under a microscope. The mean number of erythrocytes that appeared red-pink was estimated and average percentage elimination determined. Calculation of the percentage elimination of the parasites after incubation is taken as the activity of each test compound. This has been carried out using the following relation:

$$
\%=\frac{N}{N_{x}}
$$

where:

$\%=$ percentage activity of the samples,

$N=$ total number of cleared red blood cells,

$N_{\mathrm{x}}=$ total number of parasite in the red blood cells.

\section{Results and discussion}

\subsection{Chemistry}

Five monocarbonyl curcumin analogues, 3a-e were synthesized (Scheme 1) by condensation of aromatic aldehyde with acetone following standard procedure [14] with modification in the case of compounds 3a,b [10a]. The spectroscopic (IR, ${ }^{1} \mathrm{H}$ and ${ }^{13} \mathrm{C}$ NMR spectra) data of the compounds were consistent with the reported values [7, 10c, 14b, 17].
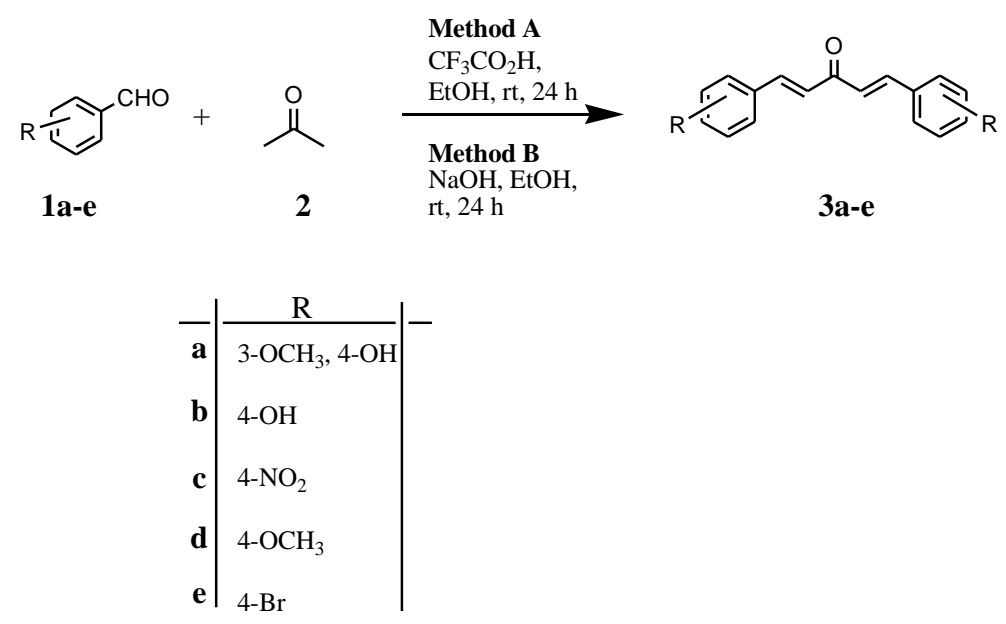

Scheme 1. Synthesis of monocarbonyl curcumin derivatives

Stereochemical configuration of the compounds was established to be $E$ based on the coupling constant (16 $\mathrm{Hz}$ ) of the two protons attached to the olefinic bond of the 1,5-diphenyl-1,4-pentadiene-3-ones.

\subsection{Antimalarial activity}

A modified method of the procedure by Hanne and coworkers was used for antimalarial activity studies [18]. The synthesized compounds were screened for in vitro antimalarial activity against the parasiteamia of $P$. falciparum using artemether drug as positive control [18], and the activity is expressed as percentage elimination of the parasites after incubation period in accordance with the reported procedure (Table 1) [19].
The preliminary structure-activity-relationship revealed that the presence of a methoxy group on the aryl ring confers strong potency on $\mathbf{3 d}$ with $57 \%$ elimination of the parasites. However introducing a hydroxyl substituent onto 3a slightly reduced the elimination potency of the parasites to $55 \%$. A further decrease in activity is observed in the case of the nitro derivative $(51 \%)$. The hydroxyl derivative, $\mathbf{3 b}$ exhibits moderate elimination potency $(48 \%)$ while the least of all the compounds appears to be the bromo substituted derivative, 3e $(42.6 \%)$. It might be inferred that all the steric and electronic factors responsible for enhancing the activity did not favor the bromo derivative. 
Table 1. Antimalarial activity of the synthesized compounds 3a-e

\begin{tabular}{ccccccc}
\hline $\mathbf{3}$ & $\begin{array}{c}\text { Initial } \\
\text { parasitized } \\
\text { count/ fields } \\
\text { before used }\end{array}$ & $\begin{array}{c}\text { Total number of } \\
\text { parasitized and } \\
\text { non-parasitized } \\
\text { fields }\end{array}$ & $\begin{array}{c}\text { Concentrations } \\
(\mathrm{mg} / \mathrm{ml})\end{array}$ & $\begin{array}{c}\text { Final count of } \\
\text { dead parasites/ } \\
\text { fields at the end } \\
\text { of incubation }\end{array}$ & $\begin{array}{c}\text { Mean of dead } \\
\text { parasites at end of } \\
\text { incubation } 72 \mathrm{~h}\end{array}$ & $\begin{array}{c}\% \text { elimination } \\
\text { at end of } \\
\text { incubation }\end{array}$ \\
\hline $\mathbf{a}$ & 88 & 116 & $10,5,2.5,1.25$ & $35+25+22+14$ & 24 & 55 \\
$\mathbf{b}$ & 88 & 116 & $10,5,2.5,1.25$ & $47+38+25+19$ & 32.25 & 48 \\
$\mathbf{c}$ & 88 & 116 & $10,5,2.5,1.25$ & $40+36+22+16$ & 28.5 & 51 \\
$\mathbf{d}$ & 88 & 116 & $10,5,2.5,1.25$ & $35+22+17+12$ & 21.5 & 57 \\
$\mathbf{e}$ & 88 & 116 & $10,5,2.5,1.25$ & $52+48+33+21$ & 38.5 & 42.6 \\
\hline
\end{tabular}

\subsection{Docking studies}

In silico exploration studies of the PfDXR active-site [20] revealed additional, adjacent binding pockets that could be explored. The presence of this additional binding pocket identified has prompted the de novo design of $N$ benzylated phosphonate esters as potential antimalarials [21].

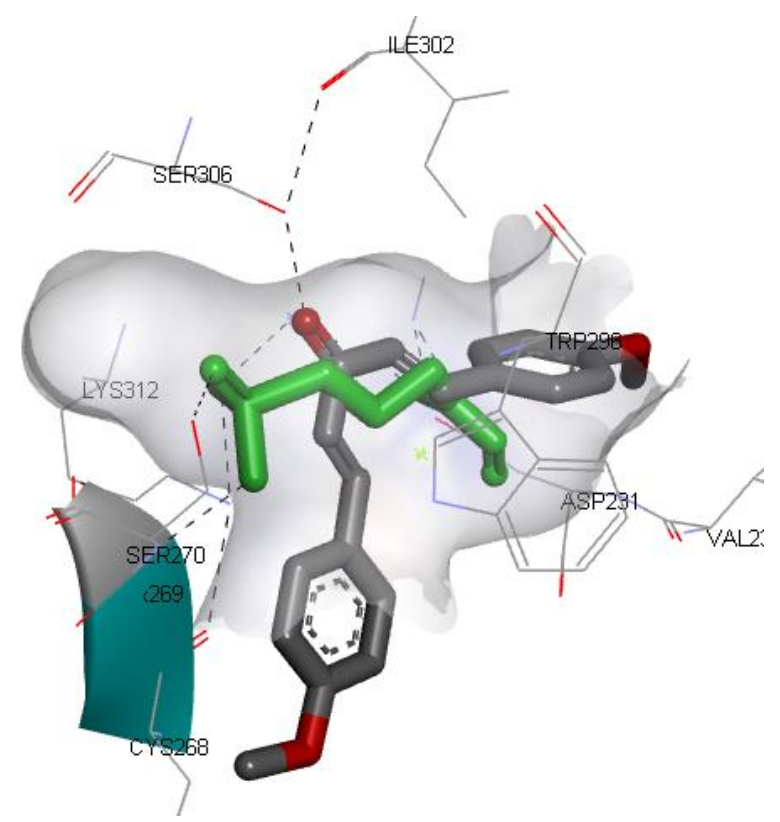

Figure 2. Docked conformations of 3d in the PfDXR receptor cavity (3AU9) [22] illustrating the close alignment of the ligand with fosmidomycin. The crystal structure conformation of fosmidomycin is shown in stick format coloured green, and the ligand in stick format coloured by atom type. Protein active-site amino acid residues are shown in wire-frame coloured by atom type. Hydrogen bonds are shown as black dashed lines.

Molecular docking plays a vital role in the rational design of drugs, by elucidating the interactions between the proposed ligands and the active-site. The different types of interactions that occur between the ligand and the active-site, can be explored and exploited to improve binding. The PfDXR crystal structure used in these modelling studies was accessed from the Research Collaboratory for Structural Bioinformatics (RCSB) protein data bank, using Discovery Studio Visualizer 4.0 [22]. Thus modelling studies were carried out in order to establish an understanding of the similarities between the synthesized compounds and fosmidomycin (a known antimalarial drug) at the PfDXR receptor cavity. To validate the reliability of the bioassay results, the protein active-site and surrounding residues were also mapped using Discovery Studio Visualizer [22]. In each case, the ligand was docked, using Autodock tools Version 4.2, into the DXR active-site after removing the water molecules present, but leaving the fosmidomycin in the PfDXR X-ray structure to note the overlap with the ligands; non-polar hydrogens were added and merged. For visual analysis, the docked ligands were overlaid with the crystal structure containing fosmidomycin within the active site.

The binding of compounds 3a-e to the PfDXR activesite revealed that all the synthesized compounds fit into the PfDXR active-site receptor cavity, exhibit potential hydrogen-bonding interactions with the proximal amino acid residues and align similar to fosmidomycin. The intrinsic feature of fosmidomycin is that its hydrophobic patch aligns parallel with the indole ring of Trp296, and each of these ligands also aligns with the indole ring. The binding also permits hydrogen-bonding interactions between the carbonyl oxygen of the ligands and Ser360 and Ile302 residues. It is noteworthy that all these compounds fill the adjacent vacant pocket (previously described) of the PfDXR active-site.

\section{Conclusion}

Five monocarbonyl curcumin analogues were synthesized and their structures identified by IR and NMR spectroscopic analysis. Their antimalarial activities were examined by measuring the percent elimination of the chloroquine-resistant parasiteamia of $P$. falciparum. Although the synthesis of these compounds along with the antimalarial activities of two of them has previously been reported, in the present study the same activity for the remaining analogues is explored for the first time. All the five compounds showed some potency compared to the known antimalarial drug, artemether. Furthermore, a molecular docking study suggests that the nature and position of the substituent play a vital role in the antimalarial activities of the compounds.

\section{Acknowledgements}

Financial support from the TETFund through the institutional-based research grant is gratefully acknowledged. The authors are thankful to Umar Sani Dorayi, Microbiology Department, Bayero University, Kano, Nigeria for the antimalarial assay. We are also indebted to Dr. Mansur Ibrahim Bala, a research fellow at the King Fahad University of Petroleum and Minerals, 
Dhahran, Saudi Arabia for the NMR spectroscopic analysis.

\section{Conflict of interest}

Authors declare no conflict of interest.

\section{References}

[1]. World Malaria Report 2014, http://www.who.int/malaria/publications/world_m alaria_report_2014/en/ (accessed December 18, 2017).

[2]. A.P. Zambre, V.M. Kulkarni, S. Padhye, S.K. Sandur and B.B. Aggarwal, Novel curcumin analogs targeting TNF-induced NF-kappaB activation and proliferation in human leukemic KBM-5 cells, Bioorg. Med. Chem. 14 (2006) 71967204.

[3]. N. Handler, W. Jaeger, H. Puschacher, K. Leisser and T. Erker, Synthesis of novel curcumin analogues and their evaluation as selective cyclooxygenase-1 (COX-1) inhibitors, Chem. Pharm. Bull. 55 (2007) 64-71.

[4]. H. Ohtsu, Z. Xiao, J. Ishida, M. Nagai, H. K. Wang, H. Itokawa, C.Y. Su, C. Shih, T. Chiang, E. Chang, Y. Lee, M.Y. Tsai, C. Chang and K.H. Lee, Antitumor Agents. 217. Curcumin Analogues as Novel Androgen Receptor Antagonists with Potential as Anti-Prostate Cancer Agents, J. Med. Chem. 45 (2002) 5037-5042.

[5]. L. Lin, Q. Shi, C.Y. Su, C.C. Shih and K.H. Lee, Antitumor agents 247. New 4-ethoxycarbonylethyl curcumin analogs as potential antiandrogenic agents, Bioorg. Med. Chem. 14 (2006) 2527-2534.

[6]. (a) R.L. Thangapazham, A. Sharma and R.K. Maheshwari, Multiple molecular targets in cancer chemoprevention by curcumin AAPS J. 8 (2006) E443; (b) R. Feng, Y. Lu, L.L. Bowman, Y. Qian, V. Castranova and M. Ding, Inhibition of Activator Protein-1, NF- $\kappa$ B, and MAPKs and Induction of Phase 2 Detoxifying Enzyme Activity by Chlorogenic Acid, J. Biol. Chem. 280 (2005) 27888-27895.

[7]. S.S Sardjiman, M.S. Reksohadiprodjo, L. Hakim, H. Goot and H. Timmerman, 1,5-Diphenyl-1,4pentadiene-3-ones and cyclic analogues as antioxidative agents. Synthesis and structureactivity relationship, Eur. J. Med. Chem. 32 (1997) 625-630.

[8]. R.A. Sharma, W.P. Steward and A.J. Gescher, Pharmacokinetics and pharmacodynamics of curcumin, Adv. Exp. Med. Biol. 595 (2007) 453 470.

[9]. R.K. Maheshwari, A.K. Singh, J. Gaddipati and R.C. Srimal, Multiple biological activities of curcumin: a short review, Life Sci. 78 (2006), $2081-$ 2087.

[10]. (a) W. Xingchuan, D. Zhi-Yun, Z. Xi, C. XiaoXing, H.C. Allan and Z. Kun, Synthesis and evaluation of curcumin-related compounds for anticancer activity, Eur. J. Med. Chem. 53 (2012) 235-245; (b) R.F. James, P. Bulbul, B. Deepak, P.E.
Jonathan, R. Nicholas, A. Dalia, L. Chenglong, L. Jiayuh and L. Pui-Kai, Structure-activity relationship studies of curcumin analogues, Bioorg. Med. Chem. Lett. 19 (2009) 2065-2069; (c) O. Hisatsugu, Y. Hiroyuki, T. Masaki, S. Masatoshi, K. Yuichi, T. Atsuko, T. Shin, K. Satoshi, S. Takao, I. Chikashi, I. Yoshiharu and S. Hiroyuki, Synthesis and biological analysis of new curcumin analogues bearing an enhanced potential for the medicinal treatment of cancer, Mol. Cancer Ther. 5 (2006) 2563-2571.

[11]. L. Guang, Y. Shulin, J. Lijuan, Z. Yu, S. Lili, X. Jian, Y. Faqing, L. Yueru and L. Xiaokun, Synthesis and Anti-bacterial Properties of Monocarbonyl Analogues of Curcumin, Chem. Pharm. Bull. 56 (2008) 162-167.

[12]. H. Lin, G. -X. Hu, J. Guo, Y. Ge, G. Liang, Q. -Q. Lian, Y. Chu, X. Yuan, P. Huang and R. - S. Ge, Mono-carbonyl curcumin analogues as $11 \beta$ hydroxysteroid dehydrogenase 1 inhibitors, Bioorg. Med. Chem. Lett. 23 (2013) 4362-4366.

[13]. Z. Fei, D. Huai-Huai, W. Yuan-Hua, W. Tian-Yi,, Y. Ze-Hao, Y. Fang, Z. Da-Zhi, C. Ying-Ying and J. Yong-Sheng, Synthesis and synergistic antifungal effects of monoketone derivatives of curcumin against fluconazole resistant Candida spp, Med. Chem. Commun. 8 (2017) 1093-1102.

[14]. (a) L. Ka-Heng, H.A. Farida, S. Ahmad, A. Faridah, S. Khozirah, A.I. Daud and H.L. Nordin, Synthesis and biological evaluation of curcumin-like diarylpentanoid analogues for anti-inflammatory, antioxidant and anti-tyrosinase activities, Eur. J. Med. Chem. 44 (2009) 3195-3200; (b) L. Guang, L. Xiaokun, C. Li, Y. Shulin, W. Xudong, S. Elaine, G. Emily, B.H. Phillip, Y. Faqing, L. Yueru and Z. Huiping, Synthesis and anti-inflammatory activities of mono-carbonyl analogues of curcumin, Bioorg. Med. Chem. Lett. 18 (2008) 1525-1529.

[15]. (a) L. Gehad, M.S. Mohamed, H.E. El Sayed, H.E. El Sayed, M.A. Yasmine, M.S. Saied and B. Assem, Synthesis, structure combined with conformational analysis, biological activities and docking studies of bis benzylidene cyclohexanone derivatives, J. Saudi Chem. Soc. 21 (2017) 619-632; (b) C. Chatchawan, P.K. Harry, I. Hasan, M.S. Sajid K.G. Matthew and S. Apichart, Curcuminoid analogs with potent activity against Trypanosoma and Leishmania species, Eur. J. Med. Chem. 45 (2010) 941-956.

[16]. B.A. Rahul, W. Gajanan, K. Neha, R.K. Ravi, K.K. Naveen, S. Dinkar and S.C. Virander, Dibenzylideneacetone analogues as novel Plasmodium falciparum inhibitors, Bioorg. Med. Chem. Lett. 21 (2011) 3034-3036.

[17]. K. Faghihi, M. Hajibeygi and M. Shabanian, Preparation and characterization of new poly(amide-imide) reinforced layer silicate nanocomposite containing N,N'-pyrromellitoylbis-1-phenyl acetic acid, J. Polym. Res. 17 (2010) 379-390.

[18]. I. Z. Hanne, S. Dan, C. Jette, H. Lars, H. Henry and W. Jerzy, In Vitro Plasmodium falciparum Drug Sensitivity Assay: Inhibition of Parasite Growth by 
Incorporation of Stomatocytogenic Amphiphiles into the Erythrocyte Membrane, J. Antimicrob. Agents Chemother. 42 (2002) 1441-1446.

[19]. M.D. Mukhtar, M. Bashir and A.H. Arzai, Comparative in-vitro studies on antiplasmodial quality of some Nigerian and foreign brands of chloroquine oral formulations marketed in kano, Afri. J. Biotechnol. 5 (2006) 2464-2468.

[20]. T. Bodill, A.C. Conibear, G.L. Blatch, K.A. Lobb, and P.T. Kaye, Synthesis and evaluation of phosphonated N-heteroarylcarboxamides as DOXP-reductoisomerase (DXR) inhibitors, Bioorg. Med. Chem. 19 (2011) 1321-1327.

[21]. C.M. Adeyemi, Faridoon, M. Isaacs, D. Mnkandhla, H.C. Hoppe, R. Krause and P.T. Kaye,
Synthesis and antimalarial activity of N-benzylated (N-arylcarbamoyl)alkylphosphonic acid derivatives, Bioorg. Med. Chem. 24 (2016) 61316138.

[22]. Dassault Systèmes BIOVIA, Discovery Studio Modelling Environment, Release 2017, San Diego: Dassault Systèmes, 2016.

Received: 12.08.2018

Received in revised form: 20.09.2018

Accepted: 22.09.2018 